\title{
Minimal Energy Path Planning for Wireless Robots
}

\author{
Chia Ching Ooi \\ Albert-Ludwigs-Universität Freiburg \\ Computer Networks and Telematics \\ Georges-Köhler-Allee 51, 79110 Freiburg, Germany \\ Email: ooi@informatik.uni-freiburg.de
}

\author{
Christian Schindelhauer \\ Albert-Ludwigs-Universität Freiburg \\ Computer Networks and Telematics \\ Georges-Köhler-Allee 51, 79110 Freiburg, Germany \\ Email: schindel@informatik.uni-freiburg.de
}

\begin{abstract}
For the first time, the problem of optimizing energy for communication and motion is investigated. We consider a single mobile robot with continuous high bandwidth wireless communication, e.g. caused by a multimedia application like video surveillance. This robot is connected to a radio base station and moves with constant speed from a given starting point on the plane to a target point. The task is to find the best path such that the energy consumption for mobility and the communication is optimized. This is motivated by the fact that the energy consumption of radio devices increases polynomially (at least to the power of two) with the transmission distance. We introduce efficient approximation algorithms finding the optimal path given the starting point, the target point and the position of the radio stations. We exemplify the influence of the communication cost by a starting scenario with one radio station. We study the performance of the proposed algorithm in simulation, compare it with the scenario without applying our approach, and present the results.
\end{abstract}

\section{INTRODUCTION}

Over the past decade, research community in the communications has been active in studying the energy efficiency of wireless communication protocols. On the other hands, researchers in robotics focus on the energy reduction for the motion planning of mobile robots. These two researches are conducted separately, and the study of networked robotics [1], [2], [3] is comparably less.

Similar to other mobile computing systems, the energy resource of most mobile robots is limited. Mobile robots have to accomplish their assigned tasks before deadlines by using the limited energy resources carried by them. The energy are meant for a number of operations: mobility, communications, performing the assigned task, computation and sensing the environment. Among them, the motion and wireless communications are two major consumers of the robot energy, apart from processing power. The overall lifetime of the robot should be maximized by efficiently distributing its energy resource.

The study of mobile ad hoc networks based on the mobile Kephera robots [4] gave us the practical insights that in certain environments, the energy consumption of communication and mobility are the two highest parameters. While the mobility cost grows linearly, the energy consumption of radio communication grows at least quadratically with the distance of two communicating robots. Therefore, at a certain range, it is advantageous to move a robot towards its communication partner. In order to achieve the goals more effectively, there are a number of challenges to judge the tradeoff of the energy usage between robot movement and communications.

In addition, the standard theoretical model for energy consumption in mobile ad hoc networks considers the distances of communication partners and the amount of data transmission, namely the flow cost model of [5]. The quadratic increase of this model is motivated by the path loss in radio communications, which can be approximated for a fixed scenario by $O\left(d^{\alpha}\right)$, where $d$ is the distance and $\alpha$ is the path loss exponent. This approximation has been established by extensive tests in several real environments leading to different path loss exponents for different environments. In [6] and [7], an additive constant is added to this term to take into account the signal processing before sending and after receiving messages.

As stated before, considering the combined energy consumption of communication and mobility of robots is new, though there is an extensive line of research for each model. One might think that mobility has only a negative impact on the behavior of wireless networks. But, recent work has shown that this is not the case. Mobility improves the coverage of wireless sensor networks [8], and helps security in ad hoc networks [9]. Furthermore, it can help with network congestion as shown in [10]. This approach overcomes the natural lower bound for throughput of $\Omega(\sqrt{n})$ by instrumenting the random movement of nodes. They design a protocol where mobile nodes relay packets and literally transport them towards the destination node. Adopting the advantages of mobility into robot communications is a new challenge that needs to be studied thoroughly.

One might assume that the communication cost is always much lower than the motion cost. In [11], the author states that the energy required for robot movements is generally much bigger than for communications. However, it is not true when the amount of data to be transmitted is very high. The communication cost should not be neglected. An example of such situation is when the overall data transmission comprises mainly the multimedia data in such applications as video streaming and surveillance. The volume of the data transmission grows as the video quality required by an application is increased. Wireless multimedia sensor network (WMSN) [12] and the mobile robot video surveillance system [13] are some of the existing applications.

Moreover, a case study [14] has shown the power breakdown of a robot, in which the motion power is not the 
component consuming the highest percentage of total energy. Instead, the embedded computer that includes the wireless communications accounts for up to $65.3 \%$ of the total energy consumption.

Our work is motivated by the applications in which a team of mobile robots has to exchange a high volume of data over the wireless medium among themselves, or to its base station during the exploration time. Thus, we concentrate on optimizing the energy consumed by both mobility and communications.

The remainder of the paper is organized as follows. In Section II, we present the system and the energy models used in our study and define the problem of computing an optimal energy path for the mobile robots. Based on these foundations, we propose approximation algorithms in Section III. The details of the simulation setup and results are then presented in Section IV, and lastly, we make concluding remarks and discuss future works.

\section{Preliminaries}

We consider a team of exploration robots and base stations that form the wireless mobile network. Each mobile robot is battery-powered and has limited lifetime. The robots and the base stations can communicate with each other through wireless transmission medium. Every robot is assigned with different task(s): searching, exploring, sensing, foraging, marking, working on target, and so on. Among them, some robots are equipped with video cameras to capture the video or pictures of the environment while they are exploring, and transmit the captured data back to the base station by either single hop or multiple hop communications.

Either the base station or the mobile robot can serve as the intermediate node for communications. At any instant, a mobile robot are assumed to know the Euclidean distance to reach its next target destination. It also knows the Euclidean distance to the base station, which serves as the destination node of the video transmission. The robot can move straight on the Euclidean path to reach its next target destination. We call this movement the Straight-Line movement in the paper. However, the Euclidean path might not be the optimal energy path since the robots consumes the energy not only for the movement but also for the wireless communications. Therefore, a minimal energy path has to be computed. We call the resulting robot movement on this optimal energy path the Smart movement.

In the following sub-sections, we introduce the system and the energy models, and define the problem of computing an optimal energy path.

\section{A. System Model}

We consider an exploration area of a mobile robot on a two dimensional Euclidean space as shown in Figure 1. A base station is located at $(0,0)$ on this exploration area. We indicate the node position as continuous function of time $p:[0, T] \mapsto \mathbb{R} \times \mathbb{R}$ such that $p(t)$ gives the position of the mobile robot at time $t$ in Cartesian Coordinates $p(t)=$

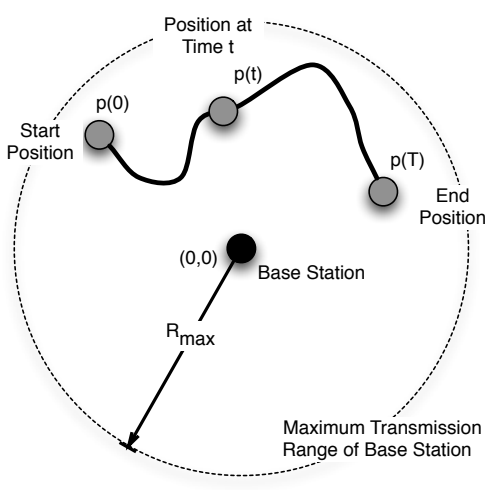

Fig. 1. Exploration Area within Communication Range

$\left(p_{x}(t), p_{y}(t)\right)$. At the beginning we have $p(0)=s$ where $s$ is the start position of the node and at the end, we have $p(T)=g$, where $g$ is the target position. When we compute the path, we approximate the path by $n$ path segments of constant speed $P=\left(\left(t_{0}, x_{0}, y_{0}\right),\left(t_{1}, x_{1}, y_{1}\right), \ldots,\left(t_{n}, x_{n}, y_{n}\right)\right)$ for $t_{0}<t_{1}<\cdots<t_{n}$ where the corresponding path function is given by

$p(t)=\left(x_{i}+\frac{\left(t-t_{i}\right)\left(x_{i+1}-x_{i}\right)}{t_{i+1}-t_{i}}, y_{i}+\frac{\left(t-t_{i}\right)\left(y_{i+1}-y_{i}\right)}{t_{i+1}-t_{i}}\right)$,

for $t \in\left[t_{i}, t_{i+1}\right)$. Let the maximum radio range of both nodes be $R_{\max }$. We limit the movement of the mobile robot to be within $R_{\max }$ so that the two nodes can communicate with each other, i.e. for all $t \in[0, T]:\|p(t)\|_{2} \leq R_{\max }$, where $\|u\|_{2}=$ $\sqrt{u_{x}^{2}+u_{y}^{2}}$ denotes the Euclidean distance to the origin. The initial position and the target position of a mobile robot must be within $R_{\max }$.

Some applications require the mobile robot to continuously transmit data at a fixed bit rate, e.g. the live video feed of a camera mounted on the mobile robot. This model is called the constant bit-rate communications model. Other applications require data transmission only at critical positions, e.g. a mobile surveillance camera in a museum which periodically transmits pictures from certain view points. We call this the position-critical communications model and illustrate it in Fig. 2. Another example application that fits both models is a robot-assisted wireless (multimedia) sensor network, in which a robot is assigned to collect the data gathered by the sensor nodes in its exploration area. Additionally, the robot may or may not be required to transmit the data collected to a base station during its exploration.

\section{B. Energy Models for Robots}

In our study, the energy model reflects two facets: energy for communication and energy for mobility. For the communications, we base the energy model on that defined in [15] and [16]. The energy required for successful wireless data transmission is affected by the distance between two communication nodes and other factors like interferences, multi-path fading, and other noises, in the transmission medium. 


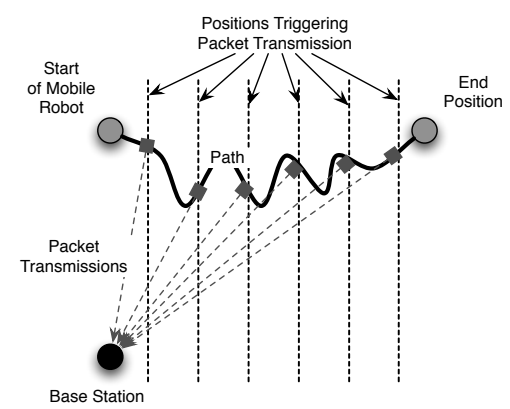

Fig. 2. Position-Critical Communications Model

TABLE I

PATH LOSS EXPONENTS FOR VARIOUS RADIO ENVIRONMENTS

\begin{tabular}{|c|c|}
\hline Environment & Path Loss Exponent, $\alpha$ \\
\hline Free Space & 2 \\
\hline Urban & 2.7 to 3.5 \\
\hline Shadowed Urban & 3 to 5 \\
\hline In-building Line of Sight & 4 to 6 \\
\hline
\end{tabular}

The energy consumed to transmit $\ell$ bits of data over the distance $d_{c}$ measured in meter is defined as:

$$
E_{t x}\left(\ell, d_{c}\right)=\ell \cdot\left(d_{c}^{\alpha} \cdot e_{t x}+e_{c c t}\right),
$$

where $e_{t x}$ is the energy required by the power amplifier of transceiver to transmit one bit data over the distance of one meter, and $e_{c c t}$ is the energy consumed in the electronic circuits of the transceiver to transmit or receive one bit, measured in the unit of Joule/bit. Depending on the transceiver sensitivity, the value of $e_{t x}$ ranges from some pico- to nanoJoule per bit per meter ${ }^{\alpha} . \alpha$ is called the path loss exponent of the transmission medium that ranges from 2 to 6 , where $\alpha \in[2,6]$ in our model.

Table I shows the path loss exponent corresponding to different types of environment in which the wireless communication takes place. In [16], it states that $\alpha=2$ and $\alpha=3,4$ is used for short and long distance or multi-path model respectively.

On the other hand, the energy consumption for receiving $\ell$ bits of data is defined as:

$$
E_{r c}(\ell)=\ell \cdot e_{c c t} .
$$

The energy consumption for receiving is independent of the distance between communicating nodes.

For the mobility, we base the energy model used in our study on that defined in [15] and [14]. The mobility energy depends on the mass of the robot, the friction to the surface (air or ground), gravity and acceleration, and the distance travelled. For simplification, we adopt the mobility energy model that is proportional to distance used in [15]. This model is reasonable for the wheeled robots [17]. It is defined as:

$$
E_{m}=m \cdot d_{m},
$$

where the movement parameter, $m$, measured in Joule/meter, is a constant based on the aforementioned factors, and $d_{m}$ is the distance traversed by the robot in meter.

a) Constant Bit-Rate Communications Model: The bitrate $B$ describes the number of bits that are sent per second. In this model, this rate is constant over the time period $[0, T]$. The total number of bits $N$ transmitted from the mobile robot to the base station is given by

$$
N=B \cdot T .
$$

Recall that the path length of a piecewise differentiable path function $p(t)$ can be described by

$$
D=\int_{t=0}^{T} \sqrt{p_{x}^{\prime 2}(t)+p_{y}^{\prime 2}(t)} \mathrm{d} t
$$

We are interested in the energy consumption of the mobile robot $E_{c b r}(p)$ consisting of transmission energy and mobility energy:

$$
E_{c b r}(p):=E_{t x}(p)+E_{m}(p),
$$

where $E_{m}(p)=m \cdot D$. For the transmission energy, we have to take into account that the mobile robot communicates while moving. This is reflected by the following term

$$
E_{t x}(p)=\int_{t=0}^{T} B \cdot\left(\|p(t)\|_{2}\right)^{\alpha} \cdot e_{t x} \mathrm{~d} t .
$$

Note that if the robot does not move, this term reduces to $B \cdot T \cdot d_{c}^{\alpha} \cdot e_{t x}$.

If the mobile robot is bounded by a maximum velocity $v_{\max }$, the following lemma shows that the best strategy is to move at maximum speed according to this model.

Lemma 1: If $B>0$ and $\|p(t)\|_{2}>0$ for $t \in[0, T]$, then for every optimal path in the constant bit-rate communications model, we have $\left\|p^{\prime}(t)\right\|_{2}=v_{\max }$ for all $t \in[0, T]$.

Proof: On the contrary, assume that $\left\|p^{\prime}(t)\right\|_{2}<v_{\max }$ for some interval $t \in\left[t_{0}, t_{1}\right]$ with $t_{0}<t_{1}$. Then we construct a new path $q(f(t))=p(t)$ using a continuous monotone increasing function $f:[0, T] \rightarrow[0, T-\delta]$, where $\delta=\left(t_{1}-t_{0}\right)-$ $\frac{1}{v_{\max }} \int_{t=t_{0}}^{t_{1}}\left\|p^{\prime}(t)\right\|_{2} \mathrm{~d} t$. Define $f(t):=t$ for $t<t_{0}, f(t):=$ $t-\delta$ for $t \in\left[t_{1}, T\right]$, and $f(t):=t_{0}+\frac{1}{v_{\max }} \int_{t=t_{0}}^{t_{1}}\left\|p^{\prime}(t)\right\|_{2} \mathrm{~d} t$ for $t \in\left[t_{0}, t_{1}\right]$.

Observe that $\left\|q^{\prime}(t)\right\|_{2} \leq v_{\max }$ for all $t \in[0, T-\delta], q(0)=$ $p(0)$, and $q(T-\delta)=p(T)$. Clearly, the mobile energy remains the same on the path $q$ since only the speed has been changed. But, the energy consumption for communication is decreased since

$$
\sum_{t=0}^{T}\left(\|p(t)\|_{2}\right)^{\alpha} \mathrm{d} t>\sum_{t=0}^{T-\delta}\left(\|q(t)\|_{2}\right)^{\alpha} \mathrm{d} t .
$$

So the path $p$ was not optimal which proves the claim.

b) Position-Critical Communications Model: In the above model, the number of transmitted bits depends on the time the robots need to reach the destination. In some application, the number of transmissions is independent of the duration of the mission, only a number of certain tasks needs to be performed, e.g. taking a picture of a certain area with a camera, measuring environmental data, etc. For these tasks, the robot needs to move to certain areas, and immediately after 
performing this task, the robot communicates the data from this area to the base station, e.g. see Fig. 2 where the mobile robot communicates a message after crossing each line.

Formally, we define a sequence of tasks (quests) $Q_{1}, \ldots, Q_{n}$ for the mobile robot, where each quest $Q_{i}=\left(A_{i}, N_{i}\right)$ consists of a region $A_{i}$ from which the robot may choose a point $p_{i} \in$ $A_{i}$ and a number of messages $N_{i}$ the robot needs to transmit after performing the task. A path of a robot solves the task $Q=\left(\left(A_{1}, N_{1}\right), \ldots,\left(A_{n}, N_{n}\right)\right)$ at points $\left(x_{1}, \ldots, x_{n}\right)$ if $\exists t_{1}<$ $t_{2}<t_{3}<\cdots<t_{n}$ with $p\left(t_{i}\right)=x_{i}$ and $x_{i} \in A_{i}$.

The energy consumption of the position-critical model for a robot with path $p$ and solution points $x=\left(x_{1}, \ldots, x_{n}\right)$ is then defined as:

$$
E_{c b r}(Q, p, x):=E_{t x}(Q, p, x)+E_{m}(p),
$$

where $E_{m}(p)=m \cdot D$ and $D$ is the path length of $p$.

$$
E_{t x}(Q, p, x)=\sum_{i=1}^{n} N_{i} \cdot\left(\left\|x_{i}\right\|_{2}\right)^{\alpha} \cdot e_{t x} .
$$

This definition can be simplified using the following lemma.

Lemma 2: For every sequence of tasks $Q$ and matching solution set $x$, a path is optimal if and only if the mobile robot moves from $x_{i}$ to $x_{i+1}$ on a straight line.

Proof: Note that for all such solutions the costs for communication energy is the same, since it depends only on the positions of $x_{i}$. Clearly the mobility energy is minimized if the mobile robot uses the straight line.

An immediate implication of this lemma is, that the speed of the robot has no influence to the energy consumption of the position-critical model. The solution set $x=\left(x_{1}, \ldots, x_{n}\right)$ gives all the necessary information for finding the optimal route and determining the energy consumption. Therefore, we refer to the position-critical energy simply by $E_{c b r}(Q, x)$.

\section{Optimal Energy Path Problems}

In the optimal energy path problem, the initial and target position of the mobile robot are given. The mobile robot communicates with the base station during its movement. The goal is to find the optimal energy path to reach the given target position.

Definition 1. The path optimization problem for positioncritical communications model.

Given a base station at $(0,0)$ and a sequence of tasks $Q=$ $\left((s, 0),\left(A_{1}, N_{1}\right), \ldots,\left(A_{n}, N_{n}\right),(g, 0)\right)$, the mobile robot has to find a (discrete) path $\left(s, x_{1}, \ldots, x_{n}, g\right)$ that solves the task, i.e. $x_{i} \in A_{i}$ for all $i \in\{1, \ldots, n\}$, and minimizes the positioncritical energy $E_{c b r}(Q, x)$.

Definition 2. The path optimization problem for constantbit-rate communications model.

Given a base station at $(0,0)$, a start position s, a target $g$, a maximal speed $v_{\max }$ and a bit-rate $B$, find a time $T$ and a path $p:[0, T] \rightarrow \mathbb{R}^{2}$ such that $p(0)=s, p(T)=g$, $\left\|p^{\prime}(t)\right\|_{2} \leq v_{\max }$ for all $t \in[0, T]$ and the bit-rate energy $E_{c b r}(p)$ is minimized.

In the following section, we describe approximation algorithms for the energy-optimal paths used in both models.

\section{Algorithms}

For the position-critical model, we have to find a solution set $x_{1}, x_{2}, \ldots, x_{n}$ such that $x_{i} \in A_{i}$, where $A_{1}$ and $A_{n}$ are single points describing the start node $s$ and the end point $g$. As a first approach, we choose a finite candidate set $V_{i, \epsilon}=y_{i, 1}, y_{i, 2}, \ldots \in A_{i}$ such that for all $u \in A_{i}$ inside the transmission range of the base station, there exists a candidate $y_{i, j}$ within distance $\epsilon$. This can be done by using a twodimensional grid positions with distances of at most $\frac{\epsilon}{\sqrt{2}}$. If the task areas are lines, then this candidate can be placed with distance $\epsilon$.

Define the edge set $E_{\epsilon}=\bigcup_{i \in\{1, \ldots, n-1\}} V_{i, \epsilon} \times V_{i+1, \epsilon}$ and the node set $V_{\epsilon}=\bigcup_{i \in\{1, \ldots, n\}} V_{i, \epsilon}$ constituting the graph $G_{\epsilon}=$ $\left(V_{\epsilon}, E_{\epsilon}\right)$.

For the edges, we define the following weight function

$$
w\left(y_{i, j}, y_{i+1, k}\right)=N_{i} \cdot\left(\left\|y_{i, j}\right\|_{2}\right)^{\alpha} \cdot e_{t x}+m \cdot\left\|y_{i, j}, y_{i+1, k}\right\|_{2}
$$

for $i<n-1$ and all $j, k$. Further, we define $w\left(y_{n-1, j}, y_{n, k}\right)=$ $\left(N_{n-1} \cdot\left(\left\|y_{n-1, j}\right\|_{2}\right)^{\alpha}+N_{n} \cdot\left(\left\|y_{n, j}\right\|_{2}\right)^{\alpha}\right) \cdot e_{t x}+m$. $\left\|y_{n-1, j}, y_{n, k}\right\|_{2}$.

Note that every path $p$ in $G_{\epsilon}$ from the start node is a valid solution of the position-critical communications model. The weight of $w(p)$ equals the energy consumption of a mobile robot on this path. Let $p_{\min }$ be the minimal energy consuming path in the original problem. By the definition of $G_{\epsilon}$, there exists a path $p$ in $G_{\epsilon}$ such that $\left\|p_{\min , i}-p_{i}\right\|_{2} \leq \epsilon$. Therefore,

$$
\left|E_{m}\left(p_{\min }\right)-E_{m}(p)\right| \leq m \cdot \epsilon \cdot(n-1) .
$$

Furthermore, one can show that

$\left|E_{t x}\left(p_{\min }\right)-E_{t x}(p)\right| \leq e_{t x} \cdot \epsilon \cdot \alpha \cdot\left(R_{\max }+\epsilon\right)^{\alpha-1} \cdot \sum_{i=1}^{n} N_{i}$,

where $R_{\max }$ is the maximum transmission distance of the base station. From this, the theorem below follows:

Theorem 1: The minimal weighted path in $G_{\epsilon}$ with respect to the weight function $w$ approximates the minimum positioncritical energy by an additive term of $m \cdot \epsilon \cdot(n-1)+e_{t x} \cdot \epsilon$. $\alpha \cdot\left(R_{\max +\epsilon}\right)^{\alpha-1} \cdot \sum_{i=1}^{n} N_{i}$.

So an approximation of the minimum energy path can be solved by using Dijkstra's shortest path algorithm. However, if the task areas $A_{i}$ are regions, i.e. containing some smallsized disk, the number of nodes of $G_{\epsilon}$ grows proportional to $\Theta\left(\frac{1}{\epsilon^{2}}\right)$ and the size of the edge set grows by $\Theta\left(\frac{1}{\epsilon^{4}}\right)$, which is the decisive term of Dijkstra's algorithm.

With the heuristic refinement strategy of Fig. 3, the running time can be considerately improved. It is an open problem whether this PCM-Dijkstra-Refinement algorithm always finds a path as good as the Dijkstra algorithm on the graph $G_{\epsilon}$, while all simulation runs show no differences for our test scenario. The following theorem shows that this algorithm is very efficient.

Theorem 2: The PCM-Dijkstra-Refinement algorithm has an asymptotic running time of $\mathcal{O}\left(n \cdot \log \left(\frac{1}{\epsilon}\right)\right)$ for general task areas aiming at an additive error bound of $\mathcal{O}(\epsilon)$. 


\section{PCM-Dijkstra-Refinement}

Carefully choose algorithm parameters $c, k>1$

$\epsilon^{\prime} \leftarrow \frac{\|s, g\|_{2}}{c}$

Construct $G_{\epsilon^{\prime}}$

Use Dijkstra's algorithm to compute optimal path $p_{\epsilon^{\prime}}$ in $G_{\epsilon^{\prime}}$

while $\epsilon^{\prime}>\epsilon$

$\epsilon^{\prime} \leftarrow \epsilon^{\prime} / c$

Refine around $p_{\epsilon^{\prime}}$ :

Construct graph $G_{\epsilon^{\prime}}$

Erase all nodes in $V_{\epsilon^{\prime}}$ which

are not within $k \cdot \epsilon^{\prime}$ distance to a node of $p$

Erase all edges adjacent to erased nodes

Use Dijkstra's algorithm to compute optimal path $p_{\epsilon^{\prime}}$ in resulting graph $G_{\epsilon^{\prime}}$

\section{end of while}

\section{return $p_{\epsilon^{\prime}}$}

Fig. 3. A Refinement Strategy for the Position-Critical Communications Model

For the constant bit-rate energy model, we use a similar approach. For $\epsilon_{2}>\epsilon_{1}>0$, define the node set $V_{\epsilon_{1}}$ such that $s, g \in V_{\epsilon_{1}}$ and for all nodes $u$ in the transmission range of the base station, there exists a node $v \in V_{\epsilon_{1}}$ with $\|u, v\|_{2} \leq \epsilon_{1}$. The edge set $E_{\epsilon_{2}}$ is defined by

$$
E_{\epsilon_{2}}=\left\{(u, v) \mid u, v \in V_{\epsilon_{1}}:\|u, v\|_{2} \leq \epsilon_{2}\right\}
$$

resulting in the graph $G_{\epsilon_{1}, \epsilon_{2}}=\left(V_{\epsilon_{1}}, E_{\epsilon_{2}}\right)$. We define the weights for the edges in $E_{\epsilon_{2}}$ as

$$
w(u, v)=B \cdot \frac{\|u, v\|_{2}}{v_{\max }} \cdot\left(\|u\|_{2}\right)^{\alpha} \cdot e_{t x}+m \cdot\|u, v\|_{2} .
$$

Theorem 3: The minimal weighted path in $G_{\epsilon_{1}, \epsilon_{2}}$ with respect to the weight function $w$ approximates the minimum bit-rate energy optimal path by a multiplicative factor of at most $1+\mathcal{O}\left(\frac{\epsilon_{1}}{\epsilon_{2}}+\epsilon_{2} \cdot\left(\frac{1}{\|s\|_{2}}+\frac{1}{\|g\|_{2}}\right)\right)$.

Proof Sketch: Consider the energy optimal path $p_{\min }$ according to the bitrate model, there exists a path $p$ in $G_{\epsilon_{1}, \epsilon_{2}}$ such that for the length $D_{\min }$ of $p_{\min }$ and the length $D$ of $p$, it holds: $\left|D-D_{\min }\right| \leq 2 \frac{\epsilon_{1}}{\epsilon_{2}} D$.

We have shown in Lemma 1 that the mobile robot moves along $p_{\min }$ with maximum speed. To approximate the communication energy, we model the number of messages to be sent between $u$ and $v$ as $B \cdot \frac{\|u, v\|_{2}}{v_{\max }}$. As the sending location, we use the starting node that leads to an error of at most $\mathcal{O}\left(\epsilon_{2}\left(\|u\|_{2}\right)^{\alpha-1} \cdot e_{t x}\right)$. If a path segment is fairer than the start point $s$ or end point $g$ from the base station, the relative error per step is smaller than $\Theta\left(\epsilon_{2} \cdot\left(\frac{1}{\|s\|_{2}}+\frac{1}{\|g\|_{2}}\right)\right)$. Otherwise, the summed error margin is small compared to the errors occuring in the vicinity of $s$ or $g$. This leads to the asymptotic bound of $\mathcal{O}\left(\epsilon_{2} \cdot\left(\frac{1}{\|s\|_{2}}+\frac{1}{\|g\|_{2}}\right)\right)$.

Using a good node placement, the graph $G_{\epsilon_{1}, \epsilon_{2}}$ has $\Theta\left(\frac{\left(R_{\max }\right)^{2}}{\left(\epsilon_{1}\right)^{2}}\right)$ nodes and $\Theta\left(\frac{\left(R_{\max }\right)^{2}\left(\epsilon_{2}\right)^{2}}{\left(\epsilon_{1}\right)^{4}}\right)$ edges. So, to achieve an approximation factor of $1 \pm \epsilon$, it is necessary to

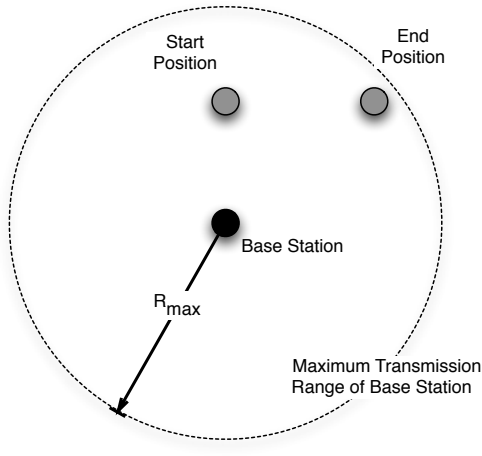

Fig. 4. Location of Mobile Robot and Base Station in Simulation

choose $\epsilon_{2}=\epsilon$ and $\epsilon_{1}=\epsilon^{2}$ which leads to a running time of the Dijkstra algorithm of $\mathcal{O}\left(\frac{1}{\epsilon^{6}}\right)$. This running time is too high for practical applications. Using the iterative refinement method in the position-critical model, it is possible to improve the running time considerably. Such algorithm (omitted due to space limitation) has a running time of $\mathcal{O}\left(\frac{1}{\epsilon^{3}}\right)$ for finding a path within the same error bound. However, the correctness of this method is yet unproven.

\section{Performance Evaluation}

In this section, we present the simulation results for both models described in Section II-A. First, the simulation setup is described. The simulation results and the analysis of the total energy consumed by the mobile robot with and without applying our proposed algorithm are presented next.

\section{A. Simulation Setup}

The mobile robot and the base station are placed in the simulation area, which is bounded by the maximum communication range between these two nodes. The data sheet of Lucent Orinoco PC card [18] for 802.11b technology shows that the communication range ranges from 25 to 550 meters varying bit rate from $1 \mathrm{Mbps}$ to $11 \mathrm{Mbps}$, for both indoor and outdoor environment. In our simulation study, the maximum communication range is configured to approximately 100 to 115 meters, which is sufficient to show the total energy saved using our proposed algorithm. A higher maximum communication range further increases the total energy saved.

Fig. 4 shows the location of the mobile robot and base station in the simulation. For simplification, the initial location of mobile robot is varied along the y-axis of the base station, while the target location is varied along the $\mathrm{x}$-axis of its initial location, up to the position that yields the maximum transmission range.

Based on the communication and the mobility energy model described in Section II-B, the simulation runs are performed according to the parameters specified in Table II. We configure the parameter $e_{c c t}, e_{t x}$ and $e_{r c}$ according to [16], and the moving parameter, $m$ to $1 \mathrm{Jm}^{-1}$, which is reasonable and realistic, as stated in [17]. As mentioned in Section II-B, the parameter $m$ is influenced by several factors including robot weight or type. The value used in the simulation is based 
TABLE II

SimULATION PARAMETERS

\begin{tabular}{|l|c|}
\hline Parameters & Values \\
\hline Video Bit Rate, $B$ (Mbps) & $1,2,3$ \\
\hline $\begin{array}{l}\text { Energy consumed by transceiver circuitry } \\
\text { to transmit or receive a bit, } e_{c c t} \text { (Joule) }\end{array}$ & $10^{-7}$ \\
\hline $\begin{array}{l}\text { Energy consumed by transceiver amplifier } \\
\text { to transmit one bit data over one meter, } e_{t x} \text { (Joule) }\end{array}$ & $10^{-12}$ \\
\hline Energy to receive a bit, $e_{r c}$ (Joule) & $10^{-7}$ \\
\hline Path Loss Exponent, $\alpha$ & 3,4 \\
\hline Energy to move robot over one meter, $m$ (Joule) & 1 \\
\hline
\end{tabular}

on the energy consumed by a wheeled robot moving on flat concrete terrain at constant friction. According to [19], a wheeled vehicle with rubber tires at one kilogram moving on concrete has to overcome $0.1 \mathrm{~N}$ force of dynamic friction. In another word, it has to expend $0.1 \mathrm{Jm}^{-1}$. Thus, our parameter $\mathrm{m}=1 \mathrm{Jm}^{-1}$ is applicable for robot up to the weight of $10 \mathrm{~kg}$. Some example wheeled robots include Khepera II at $80 \mathrm{~g}$ to $250 \mathrm{~g}$, and Khepera III at $690 \mathrm{~g}$ to $2 \mathrm{~kg}$ [20], s-bot at $660 \mathrm{~g}$ [21], and e-puck at $150 \mathrm{~g}$ [22].

As explained in Section II, path loss exponent hardly achieves 2 in realistic environment. We choose the path loss exponent of 3 and 4 as the simulation parameters. Due to the fact that the path loss exponent differs based on the environment being explored by the robot, an online path loss prediction method can be adopted by the robot to compute the resulting path loss exponent value. The simulation parameters in Table II are then varied to form a number of combination sets to further evaluate their impact on the problem.

\section{B. Simulation Results}

Based on the two models described in Section II-A, we present the results of the simulation performed according to the parameters specified in Table II. We use the terms Smart movement and Straight-Line movement to indicate the robot movement with and without applying our proposed algorithm respectively. Straight-Line movement is energy-unaware, while Smart movement is energy-aware. Once the target location of the mobile robot is selected, our algorithm is applied to the mobile robot to compute the optimal energy path.

1) Position-Critical Communications Model: We show and compare the simulation results based on Smart and StraightLine movement in Fig. 5. The total energy consumption for both Smart and Straight-Line movement are illustrated in Fig. 5(a). It also depicts the total energy saved by using the path on Smart movement. The start position of the mobile robot is 85 meters away from the base station and the target location is varied up to the maximum communication range, $R_{\max }$ predefined in our simulation setup. Using our algorithm, the total energy saved achieves $48.71 \%$. It increases when the distance between the mobile robot and base station grows. The resulting optimal path is shown in Fig. 5(b).

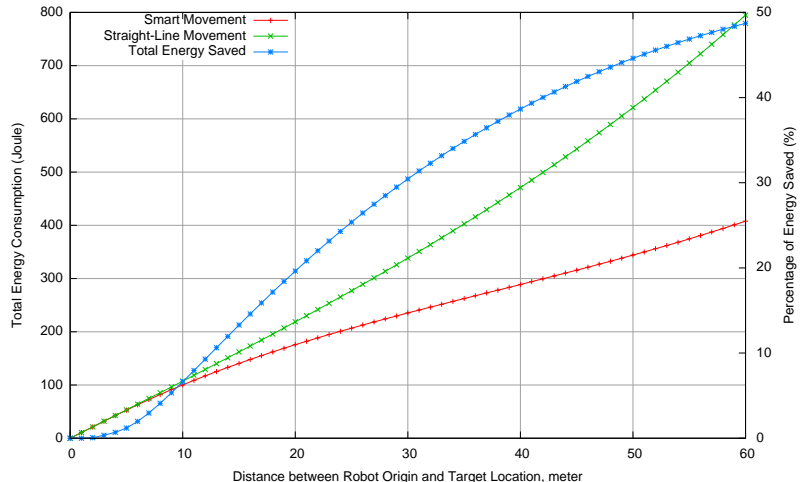

(a) Total energy consumed and percentage saved

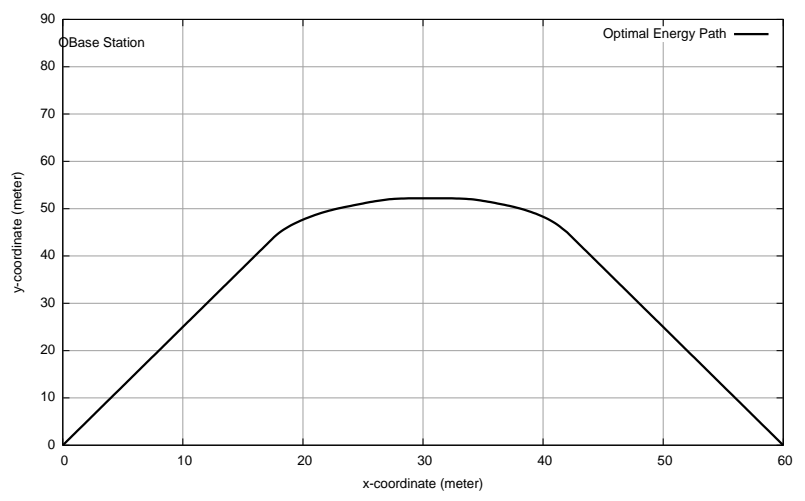

(b) Computed optimal energy path

Fig. 5. PCM: Distance between robot origin and base station $=85$ meters

For PCM model, we introduce the parameter Closeness, $c$. Each application may have its restriction on the value of Closeness parameter, depending on the size of region $A$, explained in Section II-A. A lower value of $c$ indicates the path is nearer to the path on Straight-Line movement. As this parameter limits the size of the robot exploration area, it also represents how far the optimal path is from the path on the Straight-Line movement. We vary the value of $c$ to analyze its impact on total energy savings.

For simplificity, we vary $c$ based on the precision set at 0.1 meter, where Closeness $=1$ allows the mobile robot to move downwards 0.1 meter in each step and so on. Fig. 6(a) and 6(b) illustrate the results for different Closeness allowed for the mobile robot, when the distance between the robot origin and the base station are 85 meters and 52 meters respectively. The Closeness values used in these scenarios range from 1 to 5. The results indicate that the farther a robot is allowed to move away from the Straight-Line movement, the higher the total energy is saved. Besides, it is deduced that the total energy saved tends to increase when the distance between the two communicating nodes grows.

2) Constant Bit-Rate Communications Model: In Fig. 7, we show the simulation results for the scenario when the distance between robot origin and base station is 80 meters. Fig. 7(a) and 7(b) illustrate the total energy consumption when path loss exponent is 3 and 4 respectively. The video 


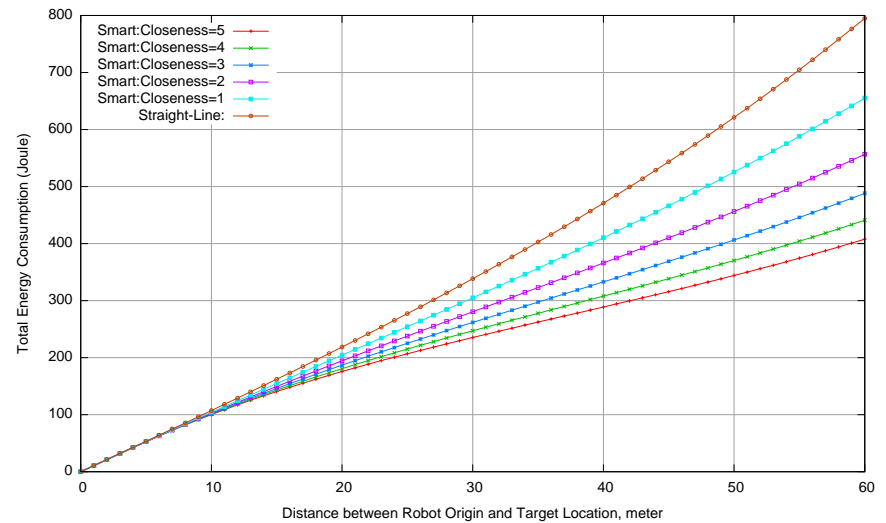

(a) Distance between robot origin and base station $=85$ meters

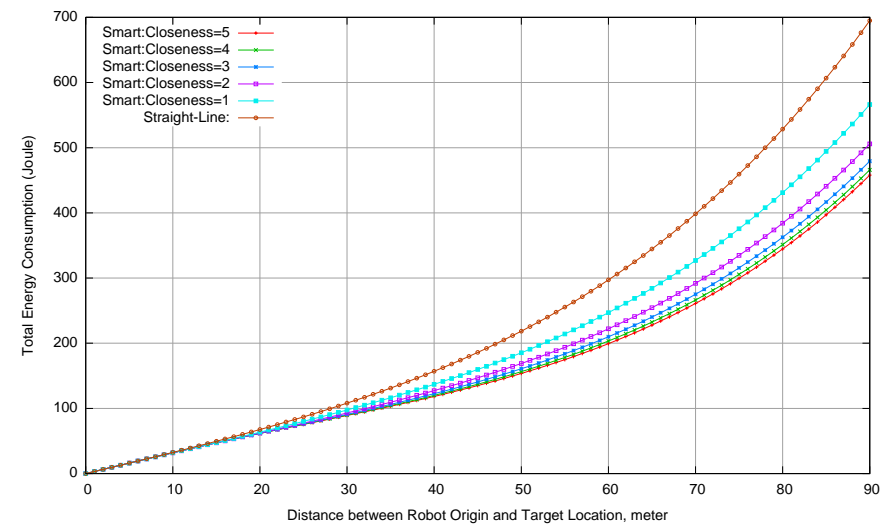

(b) Distance between robot origin and base station $=52$ meters

Fig. 6. PCM: Total Energy Consumption for varying Closeness

bitrate, $B$ ranges from 1 to $3 \mathrm{Mbps}$ in each simulation run, as indicated on the graphs. When path loss exponent is 3, the optimal energy path tends to stay on the Euclidean path in most cases, resulting in the same path computed by both Smart and Straigh-Line movements. When the video bitrate increases, the computed optimal energy path forms a curve towards the base station for larger distance between the robot origin and its target location. On the other hand, if the path loss exponent approaches 4, the optimal energy path falls on the straight line only when the distance between robot origin and its target position is below 15 meters approximately.

As the length of all the available paths computed by the the proposed algorithm varies in each scenario, and the robot moves at constant speed, total amount of video transmitted is different for each computed path. We further analyze the simulation results in this model by taking into account the length of the optimal energy path computed by both Smart and Straight-Line movement. Given the same target location, we analyze the effective energy consumption for the video transmission, $E_{\text {eff }}$, in both movements. The effective energy consumption, $E_{\text {eff }}$ is computed as:

$$
E_{\text {eff }}=E_{\text {total }} / N
$$

where $E_{\text {total }}$ is the total energy consumed by the robot to move from its origin to the target location while communicating, and

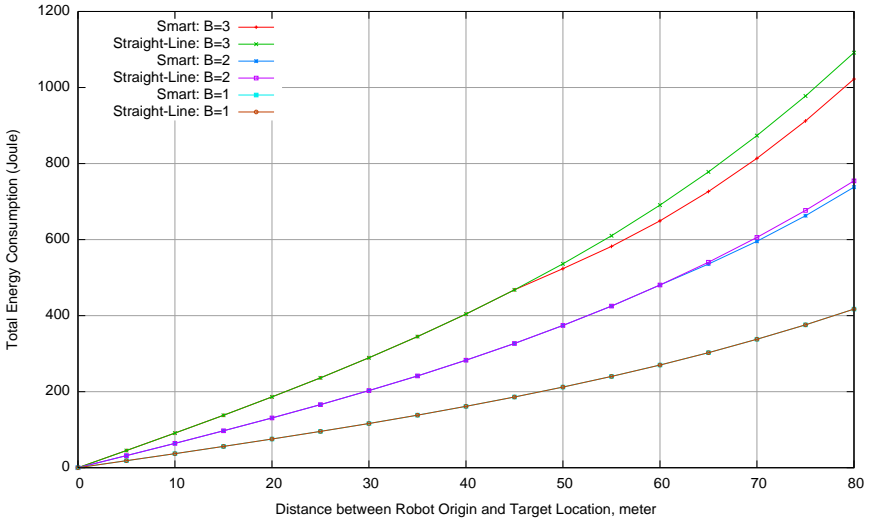

(a) Path loss exponent of 3 and varying video bitrate, $B$ (Mbps)

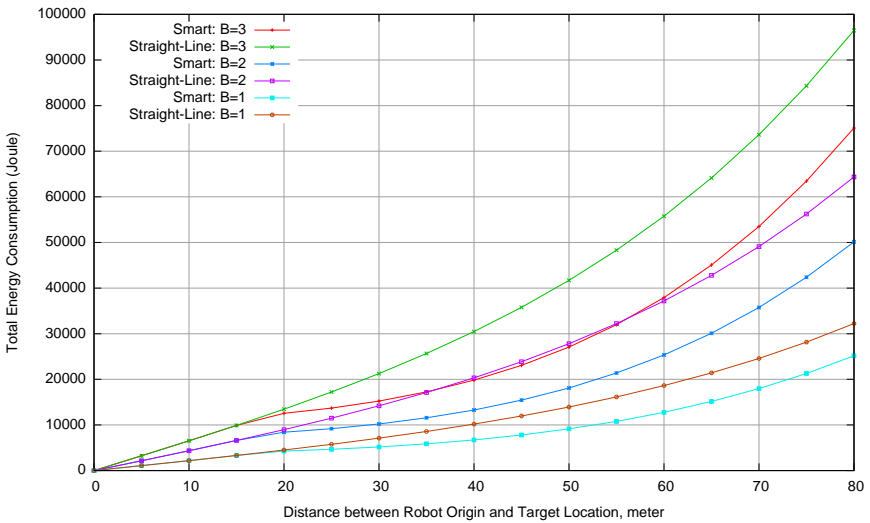

(b) Path loss exponent of 4 and varying video bitrate, $B$ (Mbps)

Fig. 7. CBR: Total energy consumption for distance between robot origin and base station $=80$ meters

$N$ indicates the total amount of bits transmitted.

Fig. 8 shows the energy efficiency of communicating mobile robot in the scenario when the distance between the robot origin and the base station is 80 meters. Fig. 8(a) and 8(b) illustrate the effective energy consumption for data transmission when path loss exponent is 3 and 4 respectively, with the video bitrate, $B$ ranging from 1 to 3 Mbps. Whenever there exist an optimal energy path computed by Smart movement that falls out of the straight line, the effective energy consumed to transmit one bit is lower than the straight line path. This improvement in energy efficiency achieves up to $70.61 \%$ in the simulation runs with $\alpha=4$ and $\mathrm{B}=3 \mathrm{Mbps}$.

Different combination of parameter values determine if the optimal energy path falls on the straight line between robot origin and its target, and the total energy saved and the robot energy efficiency using Smart movement. From our simulation, we note that the value of path loss exponent has significant impact on the computation of minimal energy path. If the exploration takes place in free space, the robot will always move on the Euclidean distance for all our simulation runs. Otherwise, its impact is influenced by other simulation parameters. Overall, the total energy saved increases with higher path loss exponent, video rate or the amount of data transmision, the distance between robot origin and its target 


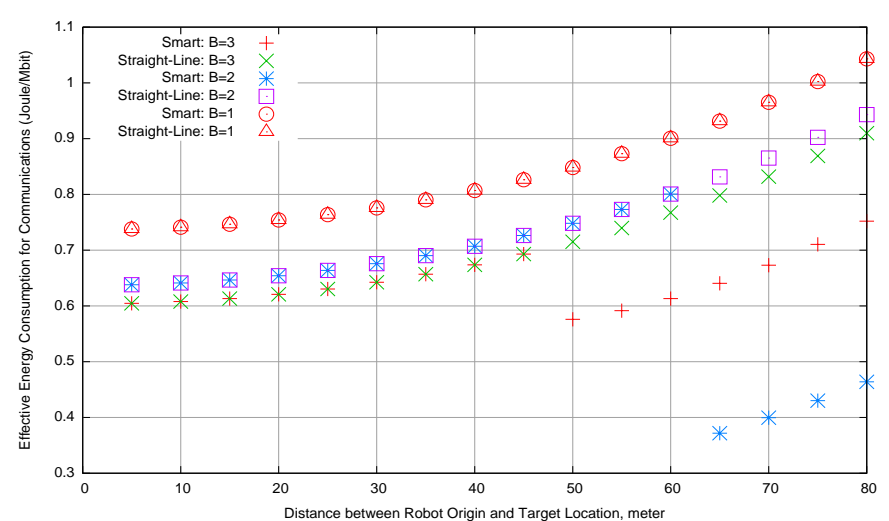

(a) Path loss exponent of 3 and varying video bitrate, $B$ (Mbps)

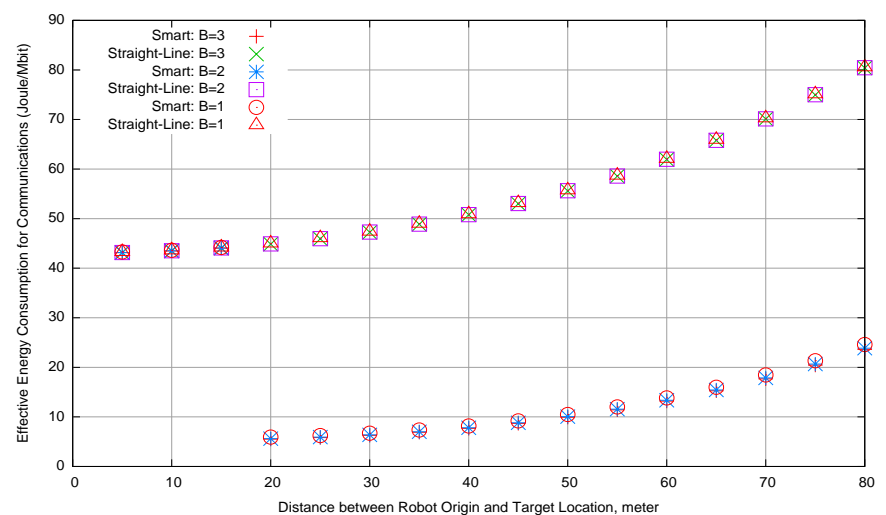

(b) Path loss exponent of 4 and varying video bitrate, $B$ (Mbps)

Fig. 8. CBR: Effective energy consumption in data transmission for distance between robot origin and base station $=80$ meters

position, as well as the distance between two communicating nodes. In contrast, it decreases over the moving parameter, which depends on the type of robot used in practice.

\section{CONCLUSions And Future Works}

In this paper, we study the problem of optimizing the energy consumption of robot that moves and communicates based on two models. An approximation algorithm based on Dijkstra's algorithm is proposed to compute the minimal energy path. We demonstrate the simulation results by applying our solution in a number of scenarios, and compare it with that of the shortest path. In the position-critical communications model, the total energy savings achieve up to nearly 50\% using the optimal path computed by our approach. In the constant bit-rate model, the optimal energy path not on the straight line movement exists in some scenarios, depending on the combination of parameter values. We show that the total energy consumption is saved up to $22.18 \%$ using our proposed solution. It also depicts that the computed path using our approach is the energy-efficient path for continuous data transmission.

Our current work involves only two communication nodes: a network of one base station and one mobile robot. Total energy savings are much higher when there are more communicating entities, for example multiple base stations, or when multihop communications are needed. Therefore, it would be interesting to find the solution of computing the optimal energy path for mobile robot communicating with multiple base stations, considering other factors such as the selection of the base station, and to study the path planning strategy for the multihop communications.

\section{ACKNOWLEDGMENT}

This research is partially supported by $D F G$ Sonderforschungsbereich SPP 1183: Organic Computing. Smart Teams: Local, Distributed Strategies for Self-Organizing Robotic Exploration Teams.

\section{REFERENCES}

[1] S. M. Das, Y. C. Hu, C. G. Lee, and Y.-H. Lu, "An efficient group communication protocol for mobile robots," in IEEE ICRA, 2005.

[2] D. Koutsonikolas, S. M. Das, Y. C. Hu, Y.-H. Lu, and C. S. G. Lee, "Cocoa: Coordinated cooperative localization for mobile multi-robot ad hoc networks," in Proceedings of the 26th IEEE ICDCSW. Washington, DC, USA: IEEE Computer Society, 2006, p. 9.

[3] M. Powers, T. Balch, and B. Lab, "Value-based communication preservation for mobile robots," in IEEE ICRA, 2004.

[4] M. Grünewald, U. Rückert, C. Schindelhauer, and K. Volbert, "Directed power-variable infrared communication for the mini robot khepera," in Proceedings of the 2nd International Conference on Autonomous Minirobots for Research and Edutainment, 2003, pp. 113-122.

[5] F. Meyer auf der Heide, C. Schindelhauer, K. Volbert, and M. Grünewald, "Congestion, dilation, and energy in radio networks," In: TOCS, vol. 37, no. 3, pp. 343-370, 2004.

[6] V. Rodoplu and T. Meng, "Minimum energy mobile wireless networks," in Proceedings of IEEE ICC, vol. 3, 1998, pp. 1633-1639.

[7] X.-Y. Li and P.-J. Wan, "Constructing minimum energy mobile wireless networks," ACM Mobile Computing and Communication Reviews, vol. 5, no. 4, pp. 55-67, 2001.

[8] B. Liu, P. Brass, O. Dousse, P. Nain, and D. Towsley, "Mobility improves coverage of sensor networks," in Proceedings of the 6th ACM MobiHoc, 2005, pp. 300-308.

[9] S. Capkun, J.-P. Hubaux, and L. Buttyán, "Mobility helps security in ad hoc networks," in Proceedings of the 4th ACM MobiHoc, 2003, pp. $46-56$.

[10] M. Grossglauser and D. N. C. Tse, "Mobility increases the capacity of ad hoc wireless networks," IEEE/ACM Trans. Netw., vol. 10, no. 4, pp. 477-486, 2002.

[11] T. H. Labella, G. Fuchs, and F. Dressler, "A simulation model for self-organised management of sensor/actuator networks," in GI/ITG KuVS Fachgespr"ach Selbstorganisierende, Adaptive, Kontextsensitive verteilte Systeme (SAKS), 2006.

[12] I. F. Akyildiz, T. Melodia, and K. R. Chowdhury, "A survey on wireless multimedia sensor networks," Comput. Networks, vol. 51, no. 4, pp. 921-960, 2007.

[13] Y.-C. Tseng, Y.-C. Wang, K.-Y. Cheng, and Y.-Y. Hsieh, "imouse: an integrated mobile surveillance and wireless sensor system," vol. 40, no. 6. IEEE Computer, 2007, pp. 60-66.

[14] H. Y. L. C. Yongguo Mei, Yung-Hsiang Lu, "A case study of mobile robot's energy consumption and conservation techniques," in 12th IEEE ICAR, 2005, pp. 492- 497.

[15] C. Tang and P. K. McKinley, "Energy optimization under informed mobility," IEEE Trans. Parallel Distrib. Syst., vol. 17, no. 9, pp. 947962, 2006.

[16] M. M. Anh Tuan Hoang, "Exploiting wireless broadcast in spatially correlated sensor networks," in IEEE ICC, 2005, pp. 2807- 2811.

[17] D. K. Goldenberg, J. Lin, A. S. Morse, B. E. Rosen, and Y. R. Yang, "Towards mobility as a network control primitive," in Proceedings of the 5th ACM MobiHoc. New York, NY, USA: ACM Press, 2004, pp. $163-174$.

[18] Orinoco PC Card, Lucent Technologies, 2000.

[19] P. A. Tipler, Physics For Scientists and Engineers. Worth Publishers, 1991.

[20] K-Team Corporation. http://www.k-team.com, 2007.

[21] Swarm-bots Project. http://www.swarm-bots.org, 2005.

[22] EPFL Education Robot. http://www.e-puck.org, 2007. 